\title{
Traducción y seguridad ciudadana
}

\author{
María del Carmen Balbuena Torezano \\ Universidad de Córdoba \\ mcbalbuena@uco.es \\ https://dx.doi.org.10.12795/futhark.2017.il2.0I
}

Fecha de recepción: 21.11.2017

Fecha de aceptación: 15.12.2017

Resumen: Este trabajo presenta las principales características un futuro proyecto de investigación interdisciplinar sobre fraseología, terminología y textos relacionados con los fenómenos relativos a los movimientos migratorios, la integración y la violencia global, cuyo objetivo fundamental es la elaboración de un sistema de información sobre el dominio del terrorismo global. En dicho sistema de información los conceptos están vinculados a una ontología, que a su vez constituye el vínculo entre términos en distintas lenguas (inglés, francés, alemán, árabe, español).

Palabras clave: flujos migratorios, terrorismo global, terminología, traducción.

\section{Translation and public safety}

Abstract: This paper focuses on the main features of an interdisciplinary research project on phraseology, terminology and texts related to phenomena that occur in migratory flows, specially on integration and international violence. The main purpose of this project is to develop an information system on international and global terrorism made of concepts linked to an ontology. It would provide terms in different languages (English, French, German, Arabic and Spanish)

Key words: migratory flows, global terrorism, terminology, translation.

Sumario: Introducción. I. Principales objetivos del proyecto y plan de trabajo. I.I. Objetivos generales. I.2. 0jetivos específicos. I.3. Fases de ejecución. 2. Resultados previsibles. Conclusiones. 


\section{Introducción}

Los grandes atentados terroristas del II/09/200I en Estados Unidos y el 1 I/03/2004 en Madrid, así como el 7/07/2005 en Londres obligaron a los estados democráticos occidentales a adoptar medidas legislativas contra el fenómeno terrorista para endurecer las penas aplicadas a este tipo de delitos. Por primera vez los gobiernos contemplan el terrorismo como un fenómeno global y complejo, desde múltiples perspectivas: cultural, política, religiosa, antropológica... en consecuencia, los fenómenos de inmigración y los movimientos migratorios, el asilo y el problema de los refugiados han sufrido igualmente variaciones jurídicas, con las consiguientes reformas restrictivas en la legislación de extranjería e inmigración.

En efecto, el principal problema que plantea esta nueva forma de terrorismo es que ya no es posible identificar claramente al enemigo, pues se trata de atentados perpetrados por grupos de rápida organización en cualquier parte del mundo, y guiados por el fanatismo religioso, el odio a occidente, la no integración en las sociedades occidentales receptoras, o, como afirman Pérez \& Gil (2015: I), "por la simple desesperación". Según datos de la EUROPOL, en 2016 I35 personas murieron a causa de un total de 13 atentados terroristas: 5 en Francia, 4 en Bélgica y 4 en Alemania, diez de los cuales fueron completados en su totalidad. Hemos de sumar a estos los lamentables ataques de Barcelona el 17/08/2017, el peor atentado yihadista perpetrado en España desde el II-M. De los 159 detenidos por pertenencia a banda armada o bajo sospechas de actividades terroristas en 20I2, la cifra ha aumentado hasta un total de 718 en 2016 en Europa.

Este nuevo escenario ha determinado, en materia investigadora, una corriente poliédrica para intentar abordar, en su conjunto, los estudios sobre inmigración, refugiados, terrorismo, y seguridad en el ámbito de la Unión Europea. Así, son numerosos los ámbitos desde los cuales se ha abordado el estudio de este problema global: trabajos sobre el concepto de terrorismo global y yihadismo (Cañete 2017; Jordán 2009; Kaldor 2003; Lobato 2017; López 2002; Milosevich 2006; Ministerio de Defensa 2005; Powell 2009, Reinares 2004 y 2008; Revilla 2005; Rodríguez et. al. 2017; Torres y Jordán 2013; Savater 2004); sobre el régimen jurídico aplicable a esta amenaza transnacional (Álvarez y González, 2006; Barrenechea, 2007; Demurtas 2014; Marica 2017; Núñez 2016); sobre seguridad, ciberseguridad y el uso de internet con fines terroristas (Álvarez \& Perdomo 2017; Guild 2015-2016; Herráiz \& Soiza 2017); sobre la protección de los derechos humanos (Consejo Internacional para el Estudio de los Derechos Humanos 2008); sobre la relación entre terrorismo, refugiados e integración (Cantón 2017; Del Valle 2016; Goig 2016; González 2015; Ibarra 2015; Knight 2017; Llorente 2016, Payero 2017; Žižek 2016); se ha tratado igualmente el tema desde el punto de vista del discurso periodístico, el lenguaje y la comunicación (Apter 2009; Bielsa 2009; Elorza 2009; García-Abadillo 2016; Lluis 2016; Bañón 2017; Ramírez 2017); los 
estudios relacionados con la terminología, la traducción o la lingüística aplicada son, sin embargo, escasos en este ámbito (Carrión 20l4; Pegenaute 20I2).

En este sentido, a la ciudadanía no le son extraños términos como "imán", "célula", "radicalización", "islamofobia", "yihadismo", "talibán" o "fundamentalismo", principalmente por su aparición en los medios informativos; sin embargo, desconocen expresiones como "terrorismo hermenéutico", "foreign fighter", "guerra híbrida", "ideología takfir", "fatua" o "doctrina Gerasinov". Parte fundamental de este discurso del terror, y de la homofobia y el rechazo que genera en la población la comunidad musulmana a consecuencia de los ataques sufridos en Europa son las figuras retóricas y las metáforas empleadas por terroristas e islamófobos, tales como "construcción estatal", "enemigo cercano", "enemigo lejano" o "cruzada contra el Estado Islámico". Consideramos que la búsqueda de esta terminología, así como las expresiones que derivan de este tipo de discursos, y su equivalencia en las lenguas europeas de los países actualmente considerados "objetivos" para llevar a cabo la yihad, resulta una herramienta necesaria para ayudar a prevenir y perseguir el terrorismo global y la islamofobia, al tiempo que proteger y preparar a la ciudadanía contra ambos fenómenos violentos.

La investigación que se llevará a cabo en el marco de este proyecto tiene como finalidad última producir un recurso multilingüe (inglés, francés, alemán, árabe, español) que reproduzca la estructura conceptual y su variación terminológica en el dominio especializado del terrorismo internacional, desde cualquiera de los ámbitos reseñados, contribuyendo con ello a la difusión del conocimiento en torno a esta compleja y poliédrica cuestión. En este sentido, pretendemos sentar las bases para el diseño de un recurso multimodal con fines de investigación y divulgación científica y social que ponga de relieve el carácter multidimensional de los conceptos propios este ámbito para la ciudadanía en general, y para educadores, fuerzas de seguridad, traductores e intérpretes en particular.

\section{Principales objetivos del proyecto y plan de trabajo}

Un trabajo de esta envergadura, y que ha de desarrollarse en distintas lenguas, debe contar con una serie de objetivos bien delimitados, entre los que cabe destacar objetivos generales y objetivos específicos.

\section{I.I. Objetivos generales}

Dos son los objetivos generales de este proyecto: 
I. Desarrollar la comunicación especializada y no especializada en torno al terrorismo global y a los flujos migratorios en un entorno multilingüe y multimodal.

2. Estudiar este léxico desde presupuestos culturales, jurídicos, antropológicos, políticos, de defensa y seguridad y lingüísticos.

\section{I.2. Objetivos específicos}

Junto a estos objetivos de carácter general, el proyecto tiene también los siguientes objetivos específicos:

I. Creación de un corpus de textos relacionados con la temática del proyecto en las lenguas de trabajo: inglés, francés, alemán, árabe, español.

2. Elaboración de un inventario de relaciones conceptuales específicas del evento [TERRORISMO], y en particular de los eventos y ontologías satélites especificando un lenguaje para las definiciones terminográficas conciso y unívoco y que permita establecer relaciones con el resto de categorías.

3. Creación de una base de datos terminológica en la que los conceptos estén vinculados a una ontología, que será el vínculo entre las distintas lenguas.

\section{I.3. Fases de ejecución}

Para la consecución de estos objetivos propuestos, es necesario llevar a cabo las siguientes fases:

- FASE I: Elaboración del inventario conceptual y del modelo de categorías y relaciones conceptuales (establecimiento de dominios y subdominios).

- FASE 2: Extracción de términos. Para ello, se realiza una búsqueda en textos especializados, divulgativos, jurídicos y humanísticos, tomando como fuentes el contenido de revistas especializadas, sitios web y publicaciones de organismos europeos, gubernamentales y no gubernamentales, organizaciones y periódicos nacionales e internacionales

- FASE 3: Elaboración de fichas de relaciones conceptuales.

- FASE 4: Creación de la base de datos multilingüe, en las lenguas de trabajo. 


\section{Resultados previsibles}

Los resultados obtenidos con este proyecto permitirán la normalización conceptual de los términos y su aproximación a la sociedad, lo que contribuirá a la reflexión profunda en torno a la integración de refugiados e inmigrantes y a la prevención de ataques islamofóbicos y de terrorismo global, no solo desde la sociedad receptora, sino también desde los propios colectivos migratorios.

Del mismo modo, el proyecto contribuirá a favorecer la tolerancia, y a prevenir la violencia contra aquellos colectivos que han sido señalados por la sociedad europea como "potencialmente peligrosos". De esta forma, será posible contribuir a la educación en valores de la ciudadanía, lo que redundará sin duda en una mayor cohesión social, cooperación y refuerzo de las medidas para impedir este tipo de delitos desde los inicios incluso de posibles actuaciones encaminadas a conseguir la captación y la radicalización.

\section{Conclusiones}

A tenor de lo expuesto en las páginas precedentes es posible establecer las siguientes conclusiones:

I. La globalización ha posibilitado el tráfico de personas de un país a otro y ha eliminado barreras, lo que supone, en principio, un gran avance desde el punto de vista humanitario y social.

2. No obstante, eso ha propiciado, igualmente, que los objetivos terroristas ya excedan de las fronteras de los países, lo que supone que, en realidad, el "objetivo global" puede estar en cualquier parte.

3. Por ello, se hace imprescindible una comunicación fluida, unívoca y eficaz entre los cuerpos de seguridad de los distintos Estados, a fin de evitar atentados terroristas y contribuir a la seguridad de los ciudadanos y ciudadanas.

4. El establecimiento de una base terminológica multilingüe contribuirá, a buen seguro, a la traducción de textos y mensajes llegados a los distintos servicios de seguridad, y facilitará de este modo la comunicación interestatal para la prevención de atentados terroristas.

5. Adicionalmente, con ello contribuiremos igualmente a la comprensión de cuestiones de tipo religioso o cultural, lo que redundará en una visión distinta del otro, más integradora e inclusiva en las sociedades de acogida. 


\section{Referencias bibliográficas}

Álvarez, L.; Perdomo, C. (2017). Estrategias de ciberseguridad nacional y ciberdefensa en la UE; retos para la estrategia de seguridad y defensa euroclatlántica. En: Durán, M. \& González, R. (Coords.), Los estudios militares y de seguridad en los albores del siglo XXI. Granada, Editorial Univ. Granada, 189-125.

Álvarez, E.; González, H. (2006). Legislación antiterrorista comparada después de los atentados del II de septiembre y su incidencia en el ejercicio de los derechos fundamentales. ARI 7/2006. [En línea: http://biblioteca.ribei.org/957///ARI-7-2006-E.pdf. Fecha de consulta: $10 / 10 / 2017]$.

Bañón, A. M. (2006). La asociación discursiva de terrorismo e inmigración. Un ejemplo de incomunicación intercultural. Revista Internacional de Comunicación Audiovisual, Publicidad y Literatura I (4), 259-277.

Barrenechea, L. (2007). ¿Están recogidas las vinculaciones entre el Derecho de Asilo y el Terrorismo en la Estrategia Global contra el Terrorismo de Naciones Unidas?. Revista Electrónica de Estudios Internacionales I4, I-10.

Cantón, J. A. (2017). Cambios en la sociedad europea como consecuencia de la crisis de los refugiados. ¿Amenaza cultural, amenaza terrorista $\circ$ política oportunista? En: Durán, M. \& González, R. (Coords.), Los estudios militares y de seguridad en los albores del siglo XXI. Granada, Editorial Univ. Granada, 67-80.

Cañete, P. (2017). El yihadismo como expresión de la violencia. Revista del Instituto Español de Estudios Estratégicos 10, I19-146.

Consejo Internacional para el Estudio de los Derechos Humanos (2008). Discusión sobre Terrorismo, Riesgos y Opciones para las Organizaciones de Derechos Humanos. Vernier (Suiza): CEIDH.

Del Valle, A. (2016). Unión Europea, crisis de refugiados y Limes Imperii. Revista General de Derecho Europeo 38. [En línea: http://rodin.uca.es/xmlui/bitstream/handle/I 0498/RGDEUR-EDITORIALCRISIS-MIGRATORIA-38-20I6-PUBLICADO.pdf?sequence=I. Fecha de consulta: $2 / 1 \mathrm{I} / 2017]$.

Elorza, A. (2007). Terrorismo y lenguaje. Cuadernos de periodistas: revista de la Asociación de Prensa de Madrid II, 10-16.

EUROPOL. (2017). Terrorism Situation and Trend Report 2017. European Union Agency for Law enforcement Cooperation.

García-Abadillo, C. (2016). El tratamiento periodístico de los atentados. Jornada de terrorismo: medios de comunicación, internet y terrorismo. Madrid, Fundación Manuel Giménez Abad, I-4.

Goig, J. M. (2016). Inmigración, asilo y refugio ante los retos actuales de la política exterior europea. Revista de Derecho UNED I8, 55-84. 
Guild, E. (20I5-20I6). Seguridad, terrorismo y asilo en el espacio Schengen. Anuario CIDOB de la Inmigración, 58-78.

Herráiz, J. A.; Soiza, N. (2017). Contraterrorismo y reorientación de las Fuerzas Armadas en misiones de seguridad interior: nueva estrategia de seguridad para una nueva forma de conflicto en el siglo XXI. En: Durán, M. \& González, R. (Coords.), Los estudios militares y de seguridad en los albores del siglo XXI. Granada, Editorial Univ. Granada, 99-I3I.

Ibarra, E. (20I5). Crisis de los refugiados, terrorismo y auge de la islamofobia. Apuntes para el debate. Cuadernos de análisis 56, 5-21.

Jordán, J. (2009). Políticas de prevención de la radicalización violencia en Europa: elementos de interés para España. Revista electrónica de Ciencia Penal y Criminología II (5). [En línea: http://criminet.ugr.es/recpc. Fecha de consulta: 29/10/2017].

Kaldor, M. (2003). Terrorismo global. Papeles 84, II-29.

Knight, K. (2017). Prácticas de exclusión de refugiados en Estados Unidos. Migraciones Forzadas 54, 52-53.

Lobato, P. (2017). ¿Existe un perfil del terrorista yihadista? En: Durán, M. \& González, R. (Coords.), Los estudios militares y de seguridad en los albores del siglo XXI. Granada, Editorial Univ. Granada, I49-163.

López, N. (2002). El concepto de terrorismo. ¿Qué terrorismo? ¿Por qué el terrorismo? ¿Hasta cuándo el terrorismo? Anuario de filosofia del derecho $|9,5|-7 \mid$.

Llorente, J. (2016). Sobre el miedo, el terrorismo, los refugiados, tú y yo. En la calle: revista sobre situaciones de riesgo social I-4.

Lluis, M. (2016). La prensa y el terrorismo. Jornada de terrorismo: medios de comunicación, internet y terrorismo. Madrid, Fundación Manuel Giménez Abad, I-9.

Marica, A. (2017). Medidas y cambios en la Unión Europea para intensificar la lucha contra el terrorismo global. Revista del Instituto Español de Estudios Estratégicos 10, 13-54.

Milosevich, M. (2006). El islam europeo: entre la integración y la radicalización. Cuadernos de pensamiento político, 209-219.

Ministerio de Defensa. IEEE. (2005). Terrorismo Internacional. Enfoques y percepciones. [En línea: http://dialnet.unirioja.es/. Fecha de consulta: $30 / 10 / 2017]$.

Payero, L. (2017). La gestión de la crisis de los refugiados en Europa: algunas reflexiones. En Uscanga, A. \& Fernández, J. A. (Dirs.), Derechos y obligaciones en el Estado de Derecho. Actas del III Coloquio binacional MéxicoEspaña, II9-132.

Pegenaute, L. (20I2). La traducción como herramienta preventiva y paliativa del terrorismo internacional. En Rabadán, R.; Guzmán, T \& Fernández, M. 
(eds.), Lengua, traducción, recepción: en honor de Julio César Santoyo. León: Universidad de León, 395-408.

Powell, Ch.; Somoza, A. (2017). La Unión Europea y la lucha contra el terrorismo global. Política Exterior 23 (127), I27-I37.

Ramírez, M. (2017). Antecedentes de la lingüística forense: ¿desde cuándo se estudia el lenguaje como evidencia? Pragmalingüística 25, 525-539.

Rodríguez, J. M.; Ceballos, M. P.; Álvarez, P.; Rey, P. (2017). Radicalización islamita: un análisis conceptual. En: Durán, M. \& González, R. (Coords.), Los estudios militares y de seguridad en los albores del siglo XXI. Granada, Editorial Univ. Granada, 29-43.

Savater, F. (2004). La lucha contra el terrorismo. Archivos del presente. Revista latinoamericana de temas internacionales 9, 23-39.

Torres, M.; Jordán, J. (20I3). Terrorismo. En Jordán, J. (Coord.), Manual de estudios estratégicos y seguridad nacional. Madrid, Plaza y Valdés, 307-328.

Žížek, S. (2016). La nueva lucha de clases. Los refugiados y el terror. Barcelona: Editorial Anagrama. 